\title{
artigo
}

Cruz, M.A.; Gomes, N.P.; Assis da Silva, K.K.; Whitaker, M.C.O.; Magalhães, J.R.F.; Santos, J.S.F.L.; Gomes, N.R.;

Crianças e adolescentes no contexto da pandemia: a interface com a violência intrafamiliar

\section{Crianças e adolescentes no contexto da pandemia: a interface com a violência intrafamiliar}

\author{
Children and adolescents in the context of the pandemic: the interface with intrafamily violence \\ Niños y adolescentes en el contexto de la pandemia: la interfaz con la violencia intrafamiliar
}

\begin{abstract}
RESUMO
Objetivo: refletir acerca da interface entre o contexto da pandemia pela Covid-19 e a vivência de violência intrafamiliar por crianças e adolescentes. Método: artigo de reflexão teórica tendo como fundamentação a literatura atual e relevante acerca da temática no que tange a violência intrafamiliar e o contexto da pandemia. Resultado: 0 estudo traz reflexões sobre a pandemia pela Covid-19 e o consequente aumento no número de casos de violência contra crianças e adolescentes, situação que já apresentava elevados índices. Além disso, se expõe fatores que têm contribuído para esse aumento, tais como o isolamento social, suspensão das aulas, sistema home office e níveis elevados de estresse devido ao contexto, o que pode guardar relação com as situações de violência. Conclusão: o estudo contribui no sentido de desvelar aspectos que alertam para a vulnerabilidade do público infanto-juvenil para experienciar abusos, oferecendo subsídios para se pensar estratégias e políticas públicas direcionadas a proteção e cuidado desse público no cenário de pandemia.
\end{abstract}

DESCRITORES: Violência doméstica; Criança; Adolescente; Pandemia; Covid-19.

\section{ABSTRACT}

Objective: to reflect on the interface between the context of the Covid-19 pandemic and the experience of intrafamily violence by children and adolescents. Method: theoretical reflection article based on the current and relevant literature on the subject with regard to intrafamily violence and the context of the pandemic. Result: The study brings reflections on the pandemic by Covid- 19 and the consequent increase in the number of cases of violence against children and adolescents, a situation that already had high rates. In addition, factors that have contributed to this increase are exposed, such as social isolation, suspension of classes, home office system and high levels of stress due to the context, which may be related to situations of violence. Conclusion: the study contributes towards unveiling aspects that alert to the vulnerability of children and adolescents to experience abuse, offering subsidies to think about strategies and public policies aimed at the protection and care of this public in the pandemic scenario.

DESCRIPTORS: Domestic violence; Kid; Teenager; Pandemic; Covid-19.

\section{RESUMEN}

Objetivo: reflexionar sobre la interfaz entre el contexto de la pandemia Covid-19 y la vivencia de violencia intrafamiliar por parte de niños y adolescentes. Método: artículo de reflexión teórica a partir de la literatura actual y relevante sobre el tema sobre la violencia intrafamiliar y el contexto de la pandemia. Resultado: El estudio trae reflexiones sobre la pandemia por Covid-19 y el consecuente incremento en el número de casos de violencia contra niños, niñas y adolescentes, situación que ya tenía altos índices. Además, se exponen factores que han contribuido a este incremento, como el aislamiento social, suspensión de clases, sistema de home office y altos niveles de estrés por el contexto, que pueden estar relacionados con situaciones de violencia. Conclusión: el estudio contribuye a desvelar aspectos que alertan sobre la vulnerabilidad de niños, niñas y adolescentes a sufrir abusos, ofreciendo subsidios para pensar estrategias y políticas públicas orientadas a la protección y atención de este público en el escenario pandémico.

DESCRIPTORES: Violencia intrafamiliar; Niño; Adolescente; Pandemia; COVID-19.

RECEBIDO EM: 22/01/2021 APROVADO EM: 16/02/2021

\section{Moniky Araújo da Cruz}

Enfermeira. Mestranda pelo Programa de Pós-graduação em Enfermagem e Saúde da Universidade Federal da Bahia. Salvador, Bahia, Brasil.

ORCID: 0000-0003-2955-5408 


\section{Nadirlene Pereira Gomes}

Enfermeira. Doutora em Enfermagem. Docente da Universidade Federal da Bahia. Salvador, Bahia, Brasil.

ORCID: 0000-0002-6043-3997

\section{Keile Kemyly Assis da Silva}

Graduanda em Enfermagem da Escola de Enfermagem da Universidade Federal da Bahia.

ORCID: 0000- 0002-1186-7502

\section{Maria Carolina Ortiz Whitaker}

Enfermeira. Doutora em Enfermagem. Docente da Universidade Federal da Bahia. Salvador, Bahia, Brasil.

ORCID: 0000-0003-0253-3831

\section{Júlia Renata Fernandes de Magalhães}

Doutora em Enfermagem. Enfermeira assistencial, UPA em Guanambi-Bahia. Coordenadora da oncologia e da unidade de internação da UNACON em Caetité, Bahia.

ORCID: 0000-0003-0631-2374

\section{Joana D'arc Ferreira Lopes Santos}

Psicóloga. Mestranda em Enfermagem pelo Programa de Pós-Graduação em Enfermagem da Universidade Federal da Bahia. Salvador, BA, Brasil.

ORCID: 0000-0001-5373-1585

\section{Nadjane Rebouças Gomes}

Enfermeira. Doutoranda em Enfermagem pelo Programa de Pós-Graduação em Enfermagem da Universidade Federal da Bahia. Salvador, BA, Brasil.

ORCID: 0000-0002-7043-3608

\section{INTRODUÇÃO}

5 rente à adoção do distanciamento social como medida preventiva de enfrentamento da pandemia pela Covid-19, a sociedade vem vivenciando o aumento no número de casos de violência contra crianças e adolescentes. Nesse cenário, onde meninas e meninos são privadas(os) do direito a uma vida livre de violência, espaços de reflexôes acerca da susceptibilidade de crianças e adolescentes à violência intrafamiliar em tempos de pandemia são essenciais no sentido de possibilitar caminhos para ações de prevenção e enfrentamento da problemática ${ }^{(1)}$.

A pandemia pela Covid-19 data do início de março de 2020, quando a Organização Mundial de Saúde (OMS) instituiu medidas para prevenção e controle da doença. Dentre elas, destaca-se o distanciamento social, cuja eficácia vem sendo reconhecida mundialmente, a exemplo de estudo realizado pela Universidade de Londres, que traz redução no número de casos da doença após a adoção do isolamento social no país ${ }^{(2)}$. Estudo nacional, desenvolvido no Ceará, corrobora acerca da eficácia desta medida ao demonstrar diminuição dos números de internações e atendimentos emergenciais depois de instaurado o distanciamento ${ }^{(3)}$.

Antes do período pandêmico, o poder dos adultos em relação às crianças e adolescentes já era exercido de maneira violenta, haja vista a naturalização dos atos. Associado a isso, os fatores estressantes sempre guardaram relação com o descontrole das mães e dos pais, ocasionando a violência.

Contudo, o distanciamento social tem favorecido a ocorrência de violência doméstica no Brasil como também em outros países. De acordo com o Ministério da Mulher, da Família e dos Direitos Humanos (MMFDH), no Brasil, considerando apenas o mês de março, houve um aumento de $18 \%$ nas denúncias relacionadas à violência registradas pelo Disque 100 e Ligue 180, ambos serviços telefônicos que prestam orientações à sociedade e realizam denúncias anônimas, sendo o primeiro acerca de violação de direitos humanos e o último voltado a violência contra mulher ${ }^{(4)}$. Estados Unidos e Reino Unido, que adotaram o distanciamento social, também reportaram aumento dos casos de violência doméstica, tendo como principais vítimas mulheres, crianças e adolescentes ${ }^{(5,6)}$. Tais dados reiteram a importância de estar atento à vulnerabilidade no âmbito doméstico.

No que diz respeito especificamente à violência contra crianças e adolescentes, órgão internacional de defesa ao público sinaliza para estimativa global de crescimento em 32\% durante a pandemia ${ }^{(7)}$. Essa realidade pode ser percebida nacionalmente a partir de 1.133 denúncias de abusos contra crianças e adolescentes contabilizadas entre 14 a 24 de março de 2020, início do isolamento social no Brasil ${ }^{(8)}$. Esse aumento, especialmente no que tange ao público infanto-juvenil, pode então guardar relação com a nova configuração nas rotinas sociais que tem intensificado a convivência entre os membros familiares, e consequentemente expõe crianças e adolescentes a ficarem confinadas em casa com mães, pais ou responsáveis. Essa preocupação encontra concordância no fato de que, conforme o Fundo das Nações Unidas para a Infância, três entre quatro crian- 
ças sofrem violência por seus pais e mães em todo o mundo ${ }^{(9)}$. Diante da realidade de confinamento das crianças e/ou adolescentes, o estudo objetiva refletir acerca da interface entre o contexto da pandemia pela Covid-19 e a vivência de violência intrafamiliar por crianças e adolescentes.

\section{MÉTODO}

Trata-se de um estudo elaborado a partir de reflexões acerca da interface entre duas temáticas: a pandemia da Covid-19 e a violência doméstica contra crianças e adolescentes. Para tanto, buscou-se artigos atuais e relevantes, de cunho nacional e internacional que fundamentaram a escrita. Após a leitura exaustiva desses materiais foi possível discorrer sobre as temáticas que versaram sobre: Distanciamento social como estratégia de enfrentamento da pandemia; Distanciamento social e aumento dos índices de violência infantil; Fatores intensificadores da violência infantil em tempos de Covid-19; Fatores precipitadores da violência infantil; e Estratégias de enfrentamento.

\section{RESULTADOS}

No contexto de distanciamento social, são diversos os fatores que se associam, tais como a ociosidade das crianças e adolescentes que ficam irritadas e a ausência ou sobrecarga de trabalho por parte das(os) responsáveis. Esses elementos estressantes confluem num cenário de permanência de todos os membros no mesmo espaço. Uma das situações que tem contribuído para a presença constante de crianças e adolescentes nos lares é a interrupção das atividades escolares. Segundo a Organização das Nações Unidas, cerca de 1,5 bilhão de crianças e adolescentes em todo o mundo estão fora da escola devido ao fechamento das instituições de ensino como iniciativa para a contenção de casos da Covid-19(10), o que tem representado maior restrição de mobilidade, distanciamento dos colegas e falta de opções de entretenimento.

O distanciamento social além de ter intensificado a permanência nos lares, limi- tou a participação nas atividades de lazer. Desde o início da pandemia, há a determinação do fechamento do comércio não essencial, como decretos de São Paulo ${ }^{(11)}$. Essa realidade também é reportada a nível internacional, a exemplo de Portugal que reduziu em $80 \%$ a mobilidade das pessoas em espaços de lazer, tais como parques, praias e praças, locais muito utilizados por crianças $^{(12)}$. Imersos nesse contexto, muitas crianças não compreendem os motivos pelo quais não podem sair de casa, situação que pode gera desgaste no processo de diálogo e negociação, culminando na violência intrafamiliar. Já as(os) adolescentes, na busca por diversão, podem descumprir a medida protetiva ao sair para encontro com amigos e demais locais, o que gera preocupação das(os) mães/pais com o contágio. Posto isso, a ausência de lazer também se configura como elemento que pode elevar ou favorecer os conflitos familiares e consequentemente a violência.

No período de pandemia, como alternativa a ausência de lazer, muitos responsáveis têm recorrido para o uso da tecnologia a fim de entreter suas(eus) filhas(os), o que tem levado a longas horas frente às telas. Todavia, pesquisa realizada na Índia, através questionários enviados eletronicamente aos responsáveis de 203 crianças, revela que $65 \%$ estão viciadas em eletrônicos, sendo a condição agravada pela pandemia ${ }^{(13)}$, além de comprometer o desenvolvimento infantil, conforme sinaliza a Sociedade Brasileira de Pediatria ${ }^{(14)}$. Contudo, salienta-se que, ao limitar o contato com telas às crianças $\mathrm{e}$ aos adolescentes, as(os) mães/pais podem passar por situações estressoras e acabar fazendo uso da violência na tentativa de controlar o uso da tecnologia.

Com relação às atividades escolares das(os) crianças/adolescentes, é importante pontuar que o próprio sistema de "Homeschooling" (educação domiciliar) demanda que mães e pais acompanhem o andamento das atividades. Esse sistema pode gerar sobrecarga e estresse para estas(es), visto que muitas(os) mães e pais não se sentem aptas(o) a realizar a condução do processo de ensino aprendizagem e apresentam dificuldades em manusear as tecnologias digitais necessárias para a realização das aulas online ${ }^{(15)}$. Tais dificuldades podem ser consideradas elementos precipitadores da violência e predispor sua ocorrência nesse tempo de pandemia.

Nesse cenário, além das demandas relacionadas ao ensino da criança, as(os) mães/pais ainda precisam lidar com as exigências do home office. Devido ao distanciamento social pesquisa, realizada com 662 empresas a fim de compreender suas ações frente à Covid-19, aponta que $98 \%$ dessas adotaram a modalidade de teletrabalho, o que gera demandas não só internas para as instituições, mas também para os funcionários ${ }^{(16)}$. Deste modo, o trabalho, que outrora era realizado com aporte tecnológico da empresa, passa a ser de responsabilidade do empregado que precisa compartilhar o material de trabalho com os(as) seus/suas filhos(as) para que realizem as atividades escolares. Essa nova configuração de necessitar organizar o revezamento dos materiais se soma ao estresse gerado pelas possíveis interrupções que os infantojuvenis podem ocasionar com a presença constante no lar, situações que podem precipitar episódios de violência.

Frente a essas mudanças, têm-se mais pessoas dentro de casa, crianças/adolescentes e mães e pais, o que resulta no aumento das atividades domésticas e consequente sobrecarga de trabalho, considerando um cenário onde trabalhadoras domésticas encontram-se afastadas. Esses afazeres dizem respeito à maior necessidade de limpeza da casa, aumento nas demandas alimentares $\mathrm{e}$, no que tange às crianças e adolescentes, maior frequência no monitoramento, ações que são, em sua maioria, atribuídas às mulheres. De acordo com estudo que discursa acerca das divisões domésticas, as mulheres ainda são as principais responsáveis pelas demandas do lar e cuidado com as(os) filhas(os), mesmo com a aproximação dos homens na realização das tarefas ${ }^{(17)}$. Essa realidade, sobretudo para as mulheres que somam a jornada de trabalho às atividades domésticas, pode elevar os conflitos familiares durante a pandemia na busca por divisões mais justas dos afazeres domiciliares, o que inclui crianças e adolescentes. Dessa 
forma, frente ao estresse da sobrecarga e/ou não realização das atividades por parte dos demais integrantes da casa, as(os) crianças/ adolescentes podem estar mais expostas a presenciar discussões ou ainda de serem vítimas de agressões.

Esse contexto provoca um cenário de circunstâncias estressoras tanto para as(os) crianças/adolescentes quanto para seus responsáveis, que também experienciam tempos difíceis. Para além das atividades domésticas e laborais demandadas para os adultos, o contexto de confinamento em família, requerido como medida de prevenção da Covid-19, perpassa por diversos marcadores sociais, tais como situação financeira, número de membros, estrutura do domicílio, possibilidades de lazer. Assim, núcleos familiares com um grande número integrantes, com baixa renda, que moram em locais precários e com poucas oportunidades de lazer podem encontrar desafios maiores para sobreviver diante do cenário atual do que as famílias de alta classe social $^{(18)}$. Um exemplo disso é estudo em Mato Grosso do Sul com famílias rurais que aponta a escassez de recursos financeiros para aquisição de equipamentos em casa, como computador e instalação de internet, mesmo antes da pandemia, necessidade que vem sendo ainda mais exigida pelas escolas nesse período ${ }^{(14)}$. Soma-se a situação de mães e pais que perderam seus empregos ou tiveram seus salários reduzidos, situações que, por si sós, já se configura como elementos disparador ou intensificador da violência.

Todos esses fatores podem predispor as crianças e os adolescentes a ficarem mais irritadas, acarretando em comportamentos agressivos ou de desobediência, exigindo maior esforço dos pais, mães e/ ou responsáveis, conforme sinaliza estudo que reflete sobre as motivações para violência contra os infantojuvenis no período de pandemia ${ }^{(18)}$. Corroborando, estudo nos Estados Unidos acerca do esgotamento parental na pandemia sinaliza para a existência de uma condição crônica chamada bournout, que consiste na elevação dos níveis de estresses por ter que lidar com as demandas de trabalho e da prole, evento associado a o que pode gerar situações de maus tratos ${ }^{(19)}$. Sendo assim, a ocorrência do estresse, angústia e ansiedade, são sentimentos que podem ser canalizados para as(os) crianças e adolescentes em forma de violência, já que muitos pais e mães descarregam suas frustrações, tensões e raiva nos filhos.

Diante tal conjuntura, se crianças e adolescentes que convivem em lares com relações saudáveis e respeitosas entre os membros da família encontram-se, em tempos de pandemia, vulneráveis à violência doméstica, essa situação é extremamente preocupante quando pensamos em crianças e adolescentes já inseridos no contexto de vulnerabilidade, como história prévia de abandono, agressão física, psicológica, sexual, entre outras formas de abusos que vitimizam o público infanto-juvenil. Estudo publicado internacionalmente faz reflexão acerca do distanciamento social e as condiçóes a ele relacionadas, as quais deixam as vítimas de violência desamparadas, devido à fragilidade das redes de atenção que são de sumária importância, sobretudo, na pandemia ${ }^{(20)}$. Assim sendo, importante refletir ainda acerca do grau de vulnerabilidade para criança sofrer violência durante a quarentena e o padrão de relacionamento previamente estabelecido com os adultos.

Vale considerar ainda que a maior predisposição infantil à vivência de violência está atrelada à desvantagem física e cognitiva das crianças e dos adolescentes em relação aos agressores, fatores que limitam o reconhecimento da situação de violência e a busca por ajuda, sobretudo, no período da quarentena. Relatório da Fiocruz ${ }^{(21)}$ que trata da interface da Covid-19 e a saúde de crianças e adolescentes enfatizam que o mesmo distanciamento social que serve de barreira para doença também limita e/ ou impede o acesso de crianças e adolescentes à rede de apoio, sejam vizinhos, familiares, escola, igreja, serviço de saúde. Fica evidente o cenário de desassistência a que estes grupos se encontram expostos nesse período de pandemia, o que requer ações que os protejam da violência intrafamiliar e assegurem o convívio familiar harmonioso e saudável durante a pandemia.
Considerando o distanciamento social, ausência da escola e de lazer que levou crianças e adolescentes a estarem ociosas em seus lares, são necessárias estratégias que as(os) entretenham, de modo a diminuir os elementos estressores. Para tanto, as famílias devem buscar atividades que possam ser realizadas no domicílio, de modo a exercer a criatividade e praticar a aprendizagem que foi comprometida com as diversas ausências.

Ressalta-se que as atividades devem promover bem-estar e manter, sempre que possível, os hábitos anteriores à pandemia, uma vez que estudo aponta que a rotina auxilia no desenvolvimento infantil por fornecer segurança ${ }^{(21)}$. Nesse sentido, os horários devem ser organizados para dar conta das aulas on-line, tarefas domésticas, atividade física, mas também contar com momentos de diversão (filmes, leituras, brincadeiras, música). Nesse ínterim, a família pode ainda realizar contato virtual com pessoas distantes (amigos e familiares), comemorar datas festivas como aniversários e festas regionais; manter e fortalecer a espiritualidade e estimular as crianças e adolescentes falarem sobre seus sentimentos, medos e desejos com vistas a diminuir a ansiedade causada pela pandemia que pode levar a situações de estresse e consequentemente situações de violência.

\section{CONCLUSÃO}

O contexto de distanciamento social, adotado com o intuito de mitigar os efeitos da pandemia pela Covid-19, vulnerabiliza crianças e adolescentes à vivência de violência intrafamiliar. Essa interface guarda relação com o confinamento doméstico, resultante do fechamento dos espaços de ensino e lazer, alterando o cotidiano de toda a família, principalmente de mães e pais que precisam lidar com questôes econômico-financeiras e com a necessidade abrupta de aprender e ensinar o manuseio das tecnologias digitais para desenvolver suas atividades laborais no cenário doméstico e, simultaneamente, assumir papéis, antes de outrem, como da escola e das empregadas domésticas. 


\section{artigo}

Cruz, M.A.; Gomes, N.P.; Assis da Silva, K.K.; Whitaker, M.C.O.; Magalhães, J.R.F.; Santos, J.S.F.L.; Gomes, N.R.;

Crianças e adolescentes no contexto da pandemia: a interface com a violência intrafamiliar

Essa nova realidade, que altera a dinâmica familiar e gera tensão e estresse, precipita e/ou intensifica relações abusivas. Diante disso, este estudo contribui no sentido de desvelar aspectos que alertam para a vulnerabilidade do público infanto-juvenil para experienciar abusos, oferecendo subsídios para se pensar estratégias e políticas públicas direcionadas a proteção e cuidado desse público no cenário de pandemia, onde, confinados em casa se é mais difícil buscar e/ou receber ajuda.

\section{REFERÊNCIAS}

1. Soares CFS e, Araújo R da S, Estrela FM, Morais AC, Farias RV, Almeida VRS et al. Fatores precipitantes e/ou agravantes da violência contra crianças no contexto da COVID-19. Brazilian J Dev [Internet]. 2021;7(1):9430-42. Available from: https:/www.brazilianjournals. com/index.php/BRJD/article/view/23714/19051

2. Mellan TA, Hoeltgebaum HH, Mishra S, Whittaker C, Schnekenberg RP, Gandy A, et al. Estimating COVID-19 cases and reproduction number in Brazil. The Preprint Server for Health Sciences. 2020;20096701. Doi: 10.1101/2020.05.09.20096701

3. Falcão J, Rabelo D, Falcão S, Pereira Neto J, Arnauld F, Belém L, et al. Impact of social isolation during COVID-19 pandemic on arrivals at emergency department and on percutaneous coronary intervention for myocardial infarction at a cardiology hospital. J Transcatheter Interv. 2020 May 27;1-4. Available from: https:// jotci.org/article/impact-of-social-isolation-during-covid-19-pandemic-on-arrivals-at-emergency-department-and-on-percutaneous-coronary-intervention-for-myocardial-infarction-at-a-cardiology-hospital/

4. Brasil. Coronavirus: sobe o número de ligações para canal de denúncia de violência doméstica na quarentena [Internet]. Brasil: Ouvidoria Nacional dos Direitos Humanos (ODNH), do Ministério da Mulher, da Familia e dos Direitos Humanos (MMFDH); 2020. Available from: https://www.gov.br/mdh/pt-br/assuntos/noticias/2020-2/marco/ coronavirus-sobe-o-numero-de-ligacoes-para-canal-de-denuncia-de-violencia-domestica-na-quarentena

5. Allen Ebrahimian, B. China's Domestic Violence Epidemic, Axios. 2020. Available from: https://www.axios.com/china domestic violencecoronavirus quarantine 7b00c3ba 35bc 4d16 afdd b76ecfb28882.html

6. Kelly J, Morgan, T. Coronavirus: Domestic abuse calls up 25\% since lockdown, charity says. BBC News. 2020. Available from: https:// www.bbc.com/news/uk-52157620

7. Huber C. A perfect storm: millions more children at risk of violence under lockdown and into the 'new normal.Equador. World Vision. 2020. Available from: https://www.wvi.org/sites/default/ files/2020-05/Aftershocks\%2OFINAL\%2OVERSION_0.pdf.

8. Childhood Brasil. A proteção de crianças e adolescentes durante 0 isolamento social. 2020. Available from: https://www.childhood.org. br/covid.

9. Fundo das Nações Unidas para a Infância (UNICEF). Violence in the lives of children and adolescentes. 2017. Available from: https://data. unicef.org/resources/a-familiar-face/

10. United Nations Educational, Scientific and Cultural Organization (UNESCO). Covid-19 educational disruption and response. 2020. Available from: https://en.unesco.org/themes/education-emergencies/coronavirus-school-closures

11. Brasil. Decreto $n^{\circ} 64.881$, de 22 de março de 2020. 2020.
Available from: https:/www.saopaulo.sp.gov.br/wp-content/uploads/2020/03/decreto-quarentena.pdf

12. Peixoto VR, Vieira A, Aguiar $P$, Sousa P, Abrantes A. Mobilidade em Portugal em tempos de pandemia por COVID-19. Escola Nacional de Saúde Pública. 2020. Available from: https://barometro-covid-19. ensp.unl.pt/wp-content/uploads/2020/04/mobilitytrends-portugal-covid-barometro-politicas-08.04.2020.pdf

13. Crescer Online. Tela x Pandemia: $65 \%$ das crianças estão viciadas em eletrônicos. 2020. Available from: https://revistacrescer.globo. com/Criancas/Comportamento/noticia/2020/07/telas-x-pandemia-65-das-criancas-estao-viciadas-em-eletronicos.html

14. Sociedade Brasileira de Pediatria (SBP). Manual de Orientação Grupo de Trabalho Saúde na Era Digital. 2019. Available from: https://www.sbp.com.br/fileadmin/user_upload/_22246c-ManOrient_-__MenosTelas__MaisSaude.pdf

15. Miranda AA, Dettmer CA, Medeiros FF. Inclusão digital: o acesso a tecnologia da informação e comunicação pelas famílias rurais do assentamento Juncal, município de Naviraí, MS. I EIGEDIN. 2017; 1(1). Available from: https:/desafioonline.ufms.br/index.php/EIGEDIN/ article/view/4407

16. Deloitte. Pesquisa: "Respostas à crise da Covid-19". 2020. Available from: http://images.e-mail.deloittecomunicacao.com. br/Web/DeloitteToucheTohmatsuAuditoresIndepende/\%7B6f3974e3-75c4-4dca-94c1-1bab0757b896\%7D_respostas-crise-covid19-Deloitte.pdf?utm_campaign=ins-052020pesquisas-covid19-download\&utm_medium=email\&utm _ source=Eloqua\&idcmp $=b r \% 3 A 2$ em $\% 3 A 3 c c \% 3 A 4$ elqbr $\% 3 A-$ 5gen\%3A6oth

17. Simao, AB. Entre o ideal e o real: percepções e práticas acerca da divisão de atividades domésticas e de cuidados no Brasil. Rev. bras. estud. popul., São Paulo. 2019;36(78). Doi: http://dx.doi. org/10.20947/s0102-3098a0078

18. Marques ES, Moraes CL, Hasselmann MH, Deslandes SF, Reichenheim ME. A violência contra mulheres, crianças e adolescentes em tempos de pandemia pela COVID-19: panorama, motivações e formas de enfrentamento. Cad.Saúde Pública. 2020;36(4). Doi: http:// dx.doi.org/10.1590/0102-311x00074420

19. Griffith AK. Parental Burnout and Child Maltreatment During the COVID-19 Pandemic. Journal of Family Violence. 2020. Available from: https:/link.springer.com/article/10.1007/s10896-020-00172-2

20. Usher KAM, Bhullar N, Durkin J, Gyamfi N, Jackson D. Family violence and COVID 19: Increased vulnerability and reduced options for support. 2020. Doi: https://doi.org/10.1111/inm.12735

21. Fundação Oswaldo Cruz (FIOCRUZ). Covid-19 e saúde da criança e do adolescente. 2020. Available from: https://www.arca.fiocruz.br/ bitstream/icict/43274/2/covid19_saude_crianca_adolescente.pdf 